\title{
A High-Voltage High-Frequency Subnanosecond Pulse Generator Based on Gallium Arsenide Drift Step-Recovery Diodes
}

\author{
A. V. Rozhkov ${ }^{a, *}$ \\ ${ }^{a}$ Ioffe Physical Technical Institute, Russian Academy of Sciences, \\ St. Petersburg, 194021 Russia \\ *e-mail:rozh@hv.ioffe.rssi.ru \\ Received February 5, 2021; revised March 3, 2021; accepted March 16, 2021
}

\begin{abstract}
The prospect of using high-voltage GaAs drift step-recovery diodes for the formation of subnanosecond pulses is shown. The electrical circuit of a generator is described, which provides (with a total efficiency of at least $25 \%$ ) the formation of pulses at a load of $50 \Omega$ with an amplitude of up to $550 \mathrm{~V}$, a voltage rise time of $0.43 \mathrm{~ns}$, a full width at half maximum of $0.73 \mathrm{~ns}$, and a repetition frequency of up to $200 \mathrm{kHz}$.
\end{abstract}

DOI: $10.1134 / \mathrm{S} 0020441221040230$

\section{INTRODUCTION}

Silicon drift step recovery diodes (DSRDs) are used to generate nanosecond high-voltage pulses in a wide switched-power range. The nanosecond recovery of the blocking ability of the DSRD semiconductor structure is provided both by the design features of the structure itself and by the parameters of the modulation mode [1]. In the developed high-voltage generators based on an assembly of several series-connected Si DSRDs, when the pulse amplitude exceeds $2 \mathrm{kV}$, the voltage rise time is at least $1.5 \mathrm{~ns}$, and the pulse FWHM duration (FWHM is the full width at halfmaximum) reaches $2-3$ ns [2, 3]. High-voltage pulse generators with an amplitude of $\sim 500 \mathrm{~V}$, a voltage rise time of $0.5-0.7 \mathrm{~ns}$, and FWHM $=(1.5-1.7) \mathrm{ns}$ are manufactured on the basis of epitaxial Si DSRDs [4, 5].

The results of the first experimental studies of GaAs-based DSRDs [6] showed a number of fundamental and constructive advantages of structures manufactured on the basis of semiconductor materials, which have a larger energy band gap compared to $\mathrm{Si}$ and a higher mobility of charge carriers. The objective of this study was to experimentally confirm the prospects of using GaAs DSRDs for generating subnanosecond high-voltage pulses.

\section{THE EXPERIMENTAL RESULTS}

Our investigation was performed using GaAs- $p^{+}-$ $p-i-n-n^{+}$structures that were manufactured using the method of liquid-phase epitaxy from a limited volume of a solution-melt of gallium arsenide in gallium. The diode structures had a distribution profile of the impurity concentration in the $p-, i-$, and $n$ regions that was characteristic of DSRDs. The concentration gradient in the base regions was as high as two orders of magnitude with a residual-impurity concentration of at most $5 \times 10^{14} \mathrm{~cm}^{-3}$ in the $i$ th region and concentrations of the acceptor and donor impurities at levels of $\sim 2 \times 10^{16} \mathrm{~cm}^{-3}$ at the interface of the $p-p^{+}$region and $\sim 5 \times 10^{15} \mathrm{~cm}^{-3}$ at the interface of the $n-n^{+}$region. The thicknesses $W$ of the $p-, i-$, and $n$ regions of the diode structures changed depending on the technological modes and were within the following ranges: $W_{p}=(20-30) \mu \mathrm{m}, W_{i}+W_{n}=(30-40) \mu \mathrm{m}$. The diode area was $\sim 0.8 \mathrm{~mm}^{2}$ and was limited to the diameter of the mesa structures, which were manufactured via chemical etching. The lifetimes of nonequilibrium charge carriers in the $n$ base $\left(\tau_{p}\right)$ that were measured by the Lax method did not exceed 100 ns. The maximum blocking diode voltage $U_{b} \sim 500 \mathrm{~V}$ was comparable with $U_{b}$ of DSRDs that were based on Si with a resistivity of $5 \Omega \mathrm{cm}$ [2]. The capacitance of GaAs DSRDs was $7-4 \mathrm{pF}$ for reverse voltages in the range of $200-$ $500 \mathrm{~V}$.

When developing the generator design, a circuitdesign solution was used to ensure an extremely high operating frequency, which allowed minimization of the heat release on a single active element of the circuit. Figure 1 shows a diagram of a push-pull generator in which the duration and amplitude of the forward $\left(J_{f}\right)$ and reverse $\left(J_{r}\right)$ current pulses were formed using two opposite-parallel $L-C$ circuits. TTL logic chips and IXDD404SIA drivers were used to generate control signals $I_{1}, I_{2}$.

The circuit operates in the following way. When a control pulse $I_{1}$ is fed to the gate of field-effect transistors $F E T_{1}$ and $F E T_{3}$ during the first oscillation halfperiod of symmetric $L_{1}-C_{1}$ and $L_{3}-C_{3}$ circuits, the 


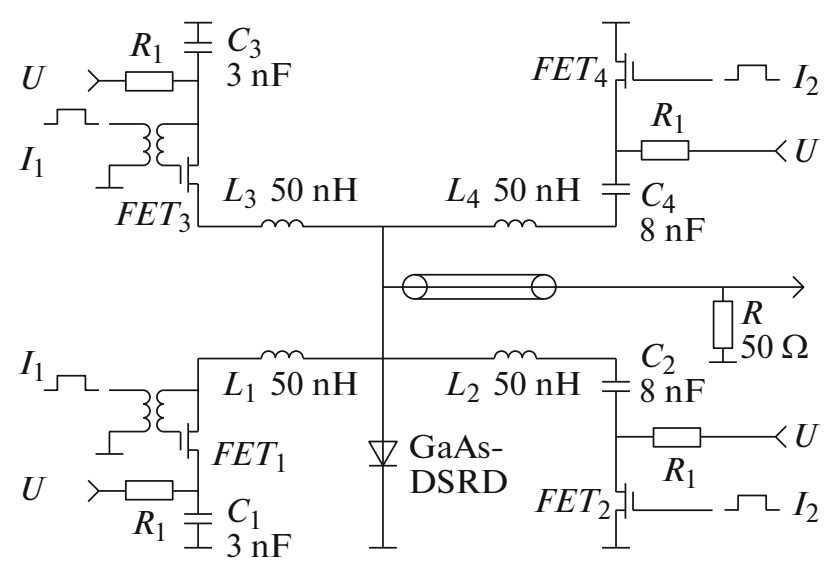

Fig. 1. The circuit diagram of the push-pull generator of high-voltage subnanosecond pulses with two oppositeparallel $L-C$ circuits: $\left(F E T_{1}-F E T_{4}\right)$ IRLML0040 fieldeffect transistors.

pumping current $J_{f}=2 J_{\max 1}$ passes through the DSRD. At the moment when the current direction changes upon application of a control pulse $I_{2}$ to the gates of the $F_{E T}$ and $F_{2} T_{4}$ FETs, the $L_{2}-C_{2}$ and $L_{4}-$ $C_{4}$ circuits of the reverse current $J_{r}=2 J_{\max 2}$ are enabled. The currents $J_{\max 1}$ and $J_{\max 2}$ are limited by the characteristic impedance of the circuits: $\rho_{1}=\left(L_{1} / C_{1}\right)^{1 / 2}=$ $\left(L_{3} / C_{3}\right)^{1 / 2}, \rho_{2}=\left(L_{2} / C_{2}\right)^{1 / 2}=\left(L_{4} / C_{4}\right)^{1 / 2}$, the resistance of the DSRD base region, and the resistance of the $F E T_{1}-F E T_{4}$ transistors. The minimum rise time of the forward and reverse currents was $t^{*}=7 \mathrm{~ns}$, which was limited by the operation time of the FETs.

In this research, the conditions for rapid recovery of the blocking ability of the DSRDs were determined, when, at a chosen rate and duration of the accumulation and dispersal of nonequilibrium charge carriers, in accordance with $[1,6]$, the necessary synchronicity of the moment of depletion of the plasma charge in the diode base and the moment of formation of the $p-n$ junction space charge region was observed. A DS06102A oscilloscope (Agilent Technologies) provided recording of the transient characteristics with a time resolution of $\sim 200$ ps. Two $20-\mathrm{dB}$ attenuators were used in the measuring path. CSV-format text files were used to save and further process the numeric data.

Figure 2 shows the oscillogram of the diode switching process until the moment of a sharp voltage increase. In the mode of the formation of subnanosecond pulses, the forward-current duration was $t_{f} \leq 20 \mathrm{~ns}$. At a voltage of $U=40 \mathrm{~V}$, the maximum value of the accumulated charge in the diode base reached $120 \mathrm{nC}$. At the specified forward-current durations, there was no diffusion modulation of the weakly doped diode base.

The thickness of the plasma diffusion layer $L_{D}=$ $\left(D t_{f}\right)^{1 / 2}$ at the values of the diffusion coefficient $D=$ $20 \mathrm{~cm}^{2} / \mathrm{s}$ that are characteristic of GaAs was at most

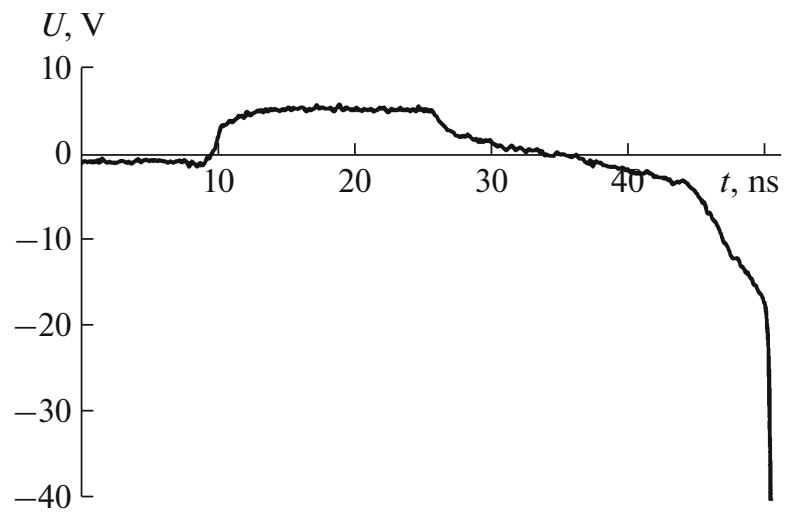

Fig. 2. A voltage waveform upon switching a GaAs-based DSRD until the moment of its sharp recovery. The power supply voltage is $U=30 \mathrm{~V}$.

$6 \mu \mathrm{m}$. The residual resistance was $\sim 1 \Omega$. The delay time of the control signal $I_{2}$ relative to $I_{1}$ was selected so that the reverse current was interrupted at the maximum of its amplitude $J_{\mathrm{r}}$. As a result of the current interruption in the DSRD, the energy that was stored in the $L_{2}$ and $L_{4}$ inductances of the reverse-current circuits was transferred within a time of $\sim L_{2} /(2 \rho)$ to a matched transmission line with the characteristic impedance $\rho=R$ and then to a load $R=50 \Omega$. In this case, the pulse amplitude across the load reached $U_{m}=$ $2 J_{\max 2} \rho$.

The oscillogram of a generator output pulse (see Fig. 3) indicates that the achieved rise time $t_{\mathrm{r}}$ and fall time $t_{\mathrm{f}}$ have record-low values for drift diodes: $t_{\mathrm{r}}=0.43 \mathrm{~ns}$ and $t_{\mathrm{f}}=0.53 \mathrm{~ns}$ for an FWHM duration of $0.72 \mathrm{~ns}$. The spread of the delay time of the output-pulse formation relative to the moment of initiation of the control pulse $I_{2}$ did not exceed the value that was set by the synchronization stability of the measuring circuit ( $\sim 30 \mathrm{ps})$. The used electrical circuit of the generator and the dynamic characteristics of the FETs provided generation of high-voltage subnanosecond pulses at frequencies of up to $200 \mathrm{kHz}$ (the pulse repetition period was $T=5 \mu \mathrm{s}$ ). At the same time, the amplitude of the voltage generated at the load was 14 times higher than the supply voltage of the generator circuit.

In the absence of radiators and air ventilation of the active circuit components, the total volume occupied by the circuit components of the push-pull generator with two opposite-parallel $L-C$ circuits was limited to dimensions of $30 \times 30 \times 50 \mathrm{~mm}$. Estimates show that the main heat release occurred at the stage of the voltage rise and fall at the active elements of the circuit. With the comparability of the total dynamic losses at the $F E T_{1}-F E T_{4}$ transistors and a single GaAs-based DSRD, the operating frequency of the generator was limited by the capabilities of the transistor switches and the value of the dissipated power in the diode structure, in which the current density reached $1.5 \mathrm{kA} / \mathrm{cm}^{2}$. 


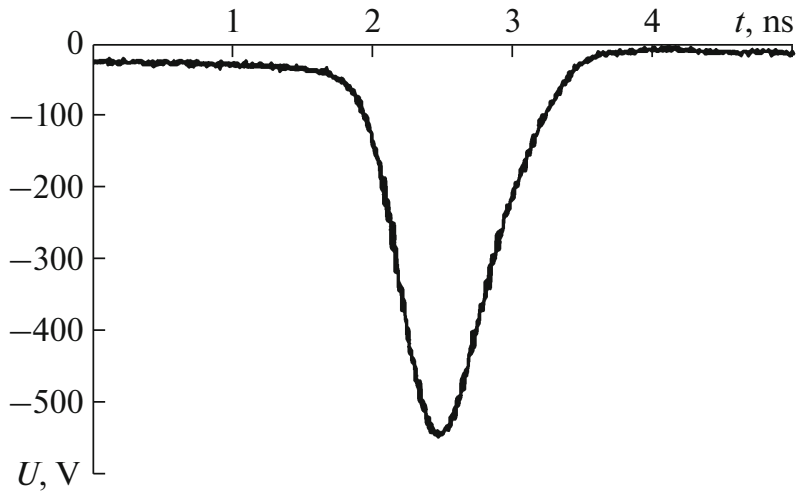

Fig. 3. A voltage waveform at the load $R=50 \Omega$. The supply voltage is $U=39 \mathrm{~V}$.

Table 1 presents the main pulse parameters of the generator as functions of the operating frequency in order to analyze the level of the achieved pulse-frequency characteristics and evaluate the modulation efficiency at higher frequencies,. The values of the voltage $U$, the average current $i_{\text {sup }}$ of the power supply, and the total efficiency $\eta$ of the generator are also given.

\section{CONCLUSIONS}

The efficiency of using GaAs-based DSRDs for generating subnanosecond high-voltage pulses at frequencies of several hundred kilohertz has been shown experimentally. It was found that the efficiency of the generator in the entire frequency range is at least $25 \%$. According to a number of parameters, the advantage of GaAs structures with a larger band gap and a higher mobility of charge carriers in comparison to Si structures has been confirmed. The current density of GaAs DSRDs is more than 10 times higher than the optimal current density of Si DSRDs. The voltage rise time and the FWHM pulse duration for GaAs DSRDs are significantly shorter than the values of the corresponding parameters for Si DSRDs. The latter provides certain advantages when using GaAs DSRDs in short-pulse modulators in ultrawideband electronics systems, since a decrease in the duration of strobe pulses leads to an increase in the overall noise immunity. The existing interest in the development of such devices determines the need for further research aimed at increasing the amplitude of output pulses in generators that are based on assemblies of several sequentially connected GaAs DSRDs.
Table 1. The main pulse parameters of the generator depending on the operating frequency

\begin{tabular}{c|c|c|c|c|c|c}
\hline$U_{m}, \mathrm{~V}$ & $t_{\mathrm{r}}, \mathrm{ns}$ & $\mathrm{FWHM}, \mathrm{ns}$ & $f, \mathrm{kHz}$ & $U, \mathrm{~V}$ & $I_{\text {sup }}, \mathrm{mA}$ & $\eta, \%$ \\
\hline 300 & 0.43 & 0.76 & 400 & 19 & 110 & 27 \\
400 & 0.43 & 0.75 & 250 & 26 & 85 & 27 \\
500 & 0.42 & 0.73 & 200 & 34 & 84 & 25 \\
\hline
\end{tabular}

\section{OPEN ACCESS}

This article is licensed under a Creative Commons Attribution 4.0 International License, which permits use, sharing, adaptation, distribution and reproduction in any medium or format, as long as you give appropriate credit to the original author(s) and the source, provide a link to the Creative Commons license, and indicate if changes were made. The images or other third party material in this article are included in the article's Creative Commons license, unless indicated otherwise in a credit line to the material. If material is not included in the article's Creative Commons license and your intended use is not permitted by statutory regulation or exceeds the permitted use, you will need to obtain permission directly from the copyright holder. To view a copy of this license, visit http://creativecommons.org/licenses/by/4.0/.

\section{REFERENCES}

1. Grekhov, I.V. and Mesyats, G.A., Usp. Fiz. Nauk, 2005, vol. 175 , no. 7 , p. 735 .

2. Voronkov, V.B., Grekhov, I.V., Kozlov, A.K., Korotkov, S.V., and Stepanyants, A.L., Instrum. Exp. Tech., 2007, vol. 50, no. 3, p. 353. https://doi.org/10.1134/S0020441207030098

3. Merensky, L.M., Kardo-Sysoev, A.F., Shmilovitch, D., and Kesar, A.S., IEEE Trans. Plasma Sci., 2013, vol. 41, no. 11, p. 3138. https://doi.org/10.1049/el.2013.2129

4. Merensky, L.M., Kesar, A.S., and Kardo-Sysoev, A.F., Proc. IEEE Int. Conference on Microwaves, Communications, Antennas and Electronic Systems (COMCAS 2013), Tel Aviv, October 21-23, 2013. 978-1-46735756-2/13.

5. Kesar, A.S., Sharabani, Y., Shafir, I., Zoran, Sh., and Sher, A., IEEE Trans. Plasma Sci., 2016, vol. 44, no. 10, p. 2424. https://doi.org/10.1109/TPS.2016.2605744

6. Rozhkov, A.V. and Kozlov, V.A., Semiconductors, 2003, vol. 37 , no. 12 , p. 1425.

Translated by A. Seferov 